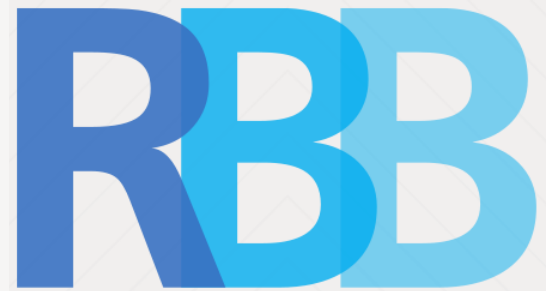

Revista Brasileira de Bioética

\begin{abstract}
Aline Albuquerque
Centro Internacional de Bioética

e Humanidades / Cátedra Unesco

de Bioética / Programa de Pós-

Graduação em Bioética da UnB,

Brasília, DF, Brasil
\end{abstract}

alineaoliveira@hotmail.com

\section{Os Direitos dos Pacientes no Brasil: análise das propostas legislativas e o papel do Sistema Único de Saúde}

\author{
Patients' Rights in Brazil: analysis of legislative proposals and the \\ role of the Brazilian System of Health
}

Resumo: O Brasil se encontra em descompasso com os demais países quando se trata da adoção de uma lei nacional de direitos dos pacientes. Este artigo tem como objetivo desenvolver aportes teóricos que fundamentam a premissa de que o Brasil deve adotar uma lei nacional de direitos dos pacientes, bem como problematizar o papel do SUS na promoção e na defesa de tais direitos. Trata-se de pesquisa de natureza teórica normativa. Concluiu-se que o endosso da necessidade de se contar com uma lei de direitos dos pacientes no Brasil correlaciona-se com a assunção pelo órgão gestor nacional do SUS de sua responsabilidade de proteger os direitos dos pacientes por meio de medidas efetivas. Reconhece-se que essa mudança propugnada corresponde a um caminho tortuoso e de longo prazo, porém, o pontapé inicial há quer ser dado pela aprovação do Projeto de Lei $n^{\circ}$ 5559/16.

Palavras-chave: paciente; direitos dos pacientes; Sistema Único de Saúde.

Abstract: Brazil is in disagreement with the other countries when it comes to the adoption of a national law on patients' rights. This article aims to develop theoretical contributions that underlie the premise that Brazil must adopt a national law on patients' rights, as well as to problematize the role of the SUS in the promotion and defense of such rights. It is a research of theoretical-normative nature. I have concluded that the endorsement of the need to have a patients 'rights law in Brazil correlates with the assumption by the national SUS management body of its responsibility to protect patients' rights through effective measures. This proposed change corresponds to a tortuous and long-term path, but the starting point is the approval of Bill 5559/16.

Keywords: patient; patient's rights; Brazilian System of Health. 


\section{Introdução}

O reconhecimento dos pacientes como titulares de direitos tão somente pelo fato de estarem sob cuidados em saúde data da década de setenta. Até esse momento histórico, os pacientes eram submetidos às prescrições dos profissionais de saúde sem serem ouvidos. Com efeito, o cuidado girava em torno da doença e a finalidade precípua dos processos terapêuticos era a cura da doença e não o alívio do sofrimento e a promoção do bem-estar do paciente. Os movimentos dos direitos civis nos Estados Unidos, alinhado ao feminista e ao dos pacientes psiquiátricos, colocaram em xeque a ideia do poder médico sobre o corpo do paciente e trouxeram à tona a obrigação ético-jurídica de respeito à sua autodeterminação. No mesmo sentido, nos anos sessenta, os movimentos do direito do consumidor abarcaram questões de saúde e desafiaram o paternalismo médico (PeledRaz, 2017).

Sendo assim, antes dos anos 70 não havia demanda pelos direitos dos pacientes (Malik, 1997). Como resultado do processo reivindicatório dos direitos dos pacientes, na década de noventa, diversos países deram início à adoção de leis específicas sobre a temática, o que prosseguiu no início do século XXI. Esse processo conjuga-se com o rechaço do paternalismo médico e o movimento na direção do cuidado centrado no paciente (Flood; May, 2012). Na Europa, os países pioneiros foram a Finlândia, a Holanda e a Hungria (European Commission, 2016); nos Estados Unidos, destaca-se a Lei de Autodeterminação do Paciente, de 1990 (United Nations, 2019), e, na América Latina, a Lei da Argentina, de 2009, que trata dos Direitos dos Pacientes, História Clínica e Consentimento Informado (Argentina, 2019); Lei do Equador, de 2006, de Amparo e Direitos dos Pacientes (Equador, 2019); Lei do Uruguai, de 2008, de Direitos e Obrigações dos Pacientes e Usuários (Uruguai, 2019). Na África, chama-se a atenção para a Carta dos Direitos dos Pacientes da África do Sul. Destaca-se, ainda a experiência de Israel, por meio da Lei dos Direitos dos Pacientes, de 1999 (Albuquerque, 2016).

O Brasil se revela em dissonância com o movimento exposto relativo à incorporação em lei dos direitos dos pacientes. Com efeito, o país não conta com uma lei nacional de direitos dos pacientes. Alguns Estados da Federação, como Minas Gerais, Rio de Janeiro, São Paulo, Brasília, Paraná, Espirito Santo e Mato Grosso adotaram leis de alcance limitado, tão somente estadual, que abarcam apenas alguns direitos dos pacientes. Não obstante se reconhecer a importância dessas leis para o avanço dos 
direitos dos pacientes no Brasil, essas leis estaduais não criaram uma consciência nacional em torno dos direitos dos pacientes, bem como não se mostraram aptas a enfrentar o paternalismo nos cuidados em saúde e a prevenir violações sofridas por pacientes nos cuidados em saúde.

Pode-se aventar uma série de fatores explicativos para o atraso brasileiro em relação à inexistência de uma lei nacional de direitos dos pacientes, dentre esses, sublinham-se: a) o paternalismo persistente que permeia a relação profissional de saúde e paciente; b) o escasso movimento da sociedade civil em torno da reivindicação de direitos dos pacientes, enquanto ativismo de direitos humanos; c) a visão consumerista da relação médico e paciente que sustenta a suficiência do Código de Defesa do Consumidor para lidar com os conflitos nos cuidados em saúde; d) a ênfase na esfera no Sistema Único de Saúde - SUS nos direitos dos usuários e a confusão entre o usuário e o paciente. Considerando que este artigo não tem como escopo analisar os fatores explicativos para a ausência no Brasil de uma lei nacional de direitos dos pacientes, sugere-se que pesquisas nesse sentido sejam realizadas.

A adoção de leis de direitos dos pacientes pressupõe o reconhecimento da importância de tais direitos e da presença de um sistema de saúde no qual os direitos dos pacientes são protegidos (Jun, 2019). Dessa forma, há que aprofundar o papel do SUS na promoção e na defesa de tais direitos, um dos escopos deste artigo.

Compreendido que o Brasil se encontra em patamar inferior quando se trata da adoção de uma lei de direitos dos pacientes, este artigo tem como objetivo desenvolver aportes teóricos que fundamentam a premissa do qual se parte, qual seja, a de que o Brasil deve adotar uma lei nacional de direitos dos pacientes, bem como analisar comparativamente o Projeto de Lei $n^{\circ}$ 5559/16 e o Projeto de Lei do Senado $\mathrm{n}^{\circ} 7$, de 2018. Ademais, tem-se como desiderato problematizar o papel do SUS na promoção e na defesa desses direitos.

Trata-se de pesquisa de natureza teórica e documental, que se alicerça nos estudos de Albuquerque (2016), Paranhos (2018), Cohen e Ezer (2013) e do Relatório da União Europeia.

Europeia sobre os Direitos dos Pacientes, de 2016 (European Commission, 2016) sobre os direitos dos pacientes, bem como na pesquisa de Jun (2019) e de Healy acerca dos efeitos positivos das leis de direitos dos pacientes (Jun, 2019). Quanto ao exame do tratamento da temática no âmbito do SUS, parte-se da análise da Consti- 
tuição Federal de 1988 e das legislações estruturantes do SUS, dos estudos de Albuquerque (2016) sobre a distinção entre usuário e paciente, bem como da pesquisa de Raz sobre saúde pública e direitos dos pacientes (Peled-Raz, 2017).

Este artigo se estrutura em três partes. A primeira diz respeito à categorização dos efeitos positivos derivados da adoção efetiva de uma lei nacional de direitos dos pacientes; a segunda versa sobre a análise das propostas legislativas: PL 5559/16 e PL do Senado 7/18 e a última trata da promoção e da proteção dos direitos dos pacientes na esfera do SUS.

\section{Categorização dos efeitos positivos corolários da ado- ção efetiva de uma lei de direitos dos pacientes}

Os direitos dos pacientes são aqueles que as pessoas têm quando se encontram sob cuidados em saúde em razão apenas do fato de serem membros da espécie humana. Conforme Albuquerque (2016), os direitos humanos dos pacientes são os seguintes: direito à vida; direito à privacidade; direito de não ser discriminado; direito à liberdade; direito à saúde; direito à informação e o direito de não ser submetido a tratamento desumano e degradante. A partir desses direitos são derivados outros mais específicos titularizados pelos pacientes.

Esses direitos se distinguem a depender do ordenamento jurídico, contudo, conforme o Relatório da União Europeia sobre os Direitos dos Pacientes, há um conjunto de direitos dos pacientes que são extraídos de diversas legislações europeias e se coadunam com o rol de tais direitos levantados na literatura (Albuquerque, 2016), a saber: direito ao consentimento informado; direito à segunda opinião; direito de recusar tratamentos e procedimentos médicos; direito de morrer com dignidade, sem sentir dor e de escolher o local de sua morte; direito à informação sobre sua condição de saúde; direito de acesso ao prontuário; direito à confidencialidade da informação pessoal; direito ao cuidado em saúde com qualidade e segurança; direito a não ser discriminado; direito de reclamar; direito à reparação e direito de participar da tomada de decisão (European Commission, 2016).

A incorporação na lei dos direitos do paciente converge para os movimentos da sociedade civil que visam ao engajamento do paciente no seu processo de cuidado e na tomada de decisão sobre questões que digam respeito ao seu corpo e à sua vida, bem como em decisões sobre alocação de recursos escassos em saúde e incorpora- 
ção de novas tecnologias em saúde ((European Commission, 2016). As leis de direitos dos pacientes fundamentam-se no cuidado pautado pela vontade e preferências do próprio paciente, apartando-se do modelo paternalista e objetiva seu empoderamento com vistas ao exercício de seus direitos. Esse modelo de cuidado em saúde derivado das leis de direitos dos pacientes ultrapassa as concepções de compaixão, de dignidade e de respeito, pois tem como escopo precípuo a reafirmação da sua condição de sujeito de direito (Health and Social Care Alliance Scotland, 2019).

No que toca aos benefícios para o paciente, familiares, profissionais e provedores de saúde derivados da adoção efetiva de uma lei de direitos dos pacientes, sublinha-se que, segundo a pesquisa de Jun, foram identificadas cinco efeitos positivos das leis de direitos dos pacientes: a) melhores serviços médicos; b) incremento da consciência profissional e da confiança na equipe de saúde; c) aumento da percepção da importância da segurança do paciente; d) declínio dos problemas legais relacionados aos médicos; e) ampliação da satisfação dos pacientes com os serviços de saúde (Jun, 2019). Considerando a formulação de Jun, serão examinados cada um dos efeitos aludidos.

A melhora dos serviços de saúde está atrelada ao fato de que uma lei de direitos dos pacientes cria um modelo de cuidado em saúde que molda políticas, programas e ações. Desse modo, tem-se o reconhecimento de que os serviços de saúde são meIhores quando as necessidades dos pacientes estão situadas no centro do processo de tomada de decisão (American Institute for Reseacher, 2019). Para tanto, os direitos dos pacientes consistem em uma forma de conferir voz ao paciente e de colocá-lo como protagonistas dos serviços de saúde. Assim, serviços de saúde avaliados como apropriados não o são apenas em razão de contar com profissionais qualificados ou competentes, mas também pelo fato de respeitarem os direitos dos pacientes, o que implica: a) a assunção pelo serviço da prerrogativa do paciente de adotar decisões sobre seu tratamento; b) a manutenção do controle pelo paciente das informações divulgadas sobre ele mesmo; c) a disponibilização sem discriminação do cuidado em saúde; d) a garantia de que os pacientes podem deixar hospitais e unidades de saúde quando desejar (Peled-Raz, 2017).

O tema da segurança do paciente é central quando se trata dos direitos dos pacientes. A correlação entre os direitos dos pacientes e a prevenção de eventos adversos nos cuidados em saúde é objeto de estudos correntes. A Joint Commission International, uma das principais instituições de acreditação do mundo, assentou 
como padrão de acreditação de hospitais o respeito aos direitos dos pacientes como meio essencial de promover a qualidade dos cuidados em saúde. Os hospitais devem informar os pacientes sobre seus direitos, como o direito à privacidade, ao consentimento e à segunda opinião. Á exemplo da Joint Commission International, a obrigação das instituições de saúde de respeitar os direitos dos pacientes significou uma mudança de paradigma nos Estados Unidos. Atualmente, grande parte dos hospitais promove os direitos dos pacientes a fim de incrementar a qualidade do cuidado em saúde (Schyve, 1997).

No que tange à informação do paciente, os cuidados em saúde são marcados pela assimetria de informação, o que impacta na sua segurança. Com efeito, as leis sobre direitos do paciente mitigam a assimetria assegurando mais informação aos pacientes, o que diminui o risco de hospitalização e de erros relacionados à medicação e aumenta a adesão ao tratamento (Healy, 2019). Desse modo, a aprovação de uma lei de direitos dos pacientes é fortemente recomendada com a finalidade de engajá-los em questões importantes relativas à sua segurança, com ênfase especial no seu direito de acesso ao prontuário (Hans Trier; Valderas; Wensing; Martin; Egebart, 2015), na medida em que ao tomar conhecimento sobre os procedimentos e os medicamentos prescritos pelo médico permite que auxilie os profissionais de saúde a adotar medidas de prevenção de eventos adversos.

A diminuição da responsabilização jurídica dos profissionais de medicina é um fator relevante comumente associado ao fato do país contar com uma lei de direitos dos pacientes. A repercussão na redução da judicialização da medicina se correlaciona com o incremento do nível de satisfação dos pacientes e com a sua participação no processo de tomada de decisão (Health and Social Care Alliance Scotland, 2019). Os direitos dos pacientes são instrumentos de promoção da educação das equipes de saúde, conseguintemente, equipes que respeitam os direitos dos pacientes apresentam maior capacidade de se comunicar com o paciente e de satisfazê-lo (Schyve , 1997). Ademais, as leis de direitos dos pacientes devem ser efetivadas mediante a implantação de mecanismos extrajudiciais de solução de conflitos. Por exemplo, Ombudsman do Paciente e outros serviços destinados ao recebimento e ao processamento de reclamações de pacientes decorrem das leis sobre os direitos dos pacientes e reduzem a judicialização e a punição de médicos, por exemplo, na Nova Zelândia, passou-se de 84 processos disciplinares contra médicos, em 1996, para 8 em 2010 (Flood; May, 2012). 
Quando os pacientes têm seus direitos respeitados, conseguintemente, sentem-se mais satisfeitos com os serviços de saúde. As leis sobre direitos dos pacientes fomentam o modelo do cuidado centrado no paciente, criando uma nova cultura nos serviços de saúde que se alicerça na parceria entre o paciente e o profissional, o que resulta em melhores condições de saúde para o paciente (Healy, 2019). Desse modo, as leis de direitos dos pacientes são compreendidas como uma ferramenta central na mitigação da assimetria de poder e de conhecimento que permeia a relação profissional de saúde e paciente, estimulando, assim, a parceria entre ambos (European Commission, 2016).

As leis de direitos dos pacientes têm o condão de impulsionar o incremento da consciência do profissional de saúde acerca da essencialidade de se colocar o paciente no centro do cuidados, e da confiança do paciente na equipe de saúde. Nesse sentido, a fidúcia e a responsividade dos serviços de saúde que são derivadas das leis de direitos dos pacientes impactam positivamente na satisfação do paciente (Meesala; Paul, 2018).

Como visto, pesquisas apontam para a presença de efeitos positivos corolários da adoção efetiva das leis de direitos dos pacientes para os próprios pacientes, bem como para profissionais e provedores de serviços de saúde. Embora a investigação sobre tais efeitos seja escassa e, no Brasil, inexistente em razão da ausência de uma lei nacional de direitos dos pacientes, pode-se depreender dos estudos apontados que as leis de direitos do paciente contribuem para a melhora da qualidade dos serviços de saúde, a diminuição da judicialização da medicina e o incremento da satisfação do paciente. Desse modo, não obstante as pesquisas que apontam para os benefícios advindos da adoção de uma lei nacional de direitos dos pacientes, a efetivação dos direitos dos pacientes, mesmo na Europa que conta com um arcabouço legislativo desde o início da década de noventa, ainda permanece um desafio.

A dificuldade de se assegurar na prática tais direitos é atribuída ao fato de que os direitos dos pacientes são desconhecidos da ampla maioria das pessoas e a percepção da sua relevância ainda é baixa entre os próprios pacientes, familiares, profissionais e provedores de serviços de saúde. Esse fato se conjuga com o paternalismo médico que ainda predomina nas relações de cuidados em saúde (European Commission, 2016). Esse panorama foi constatado na Europa a partir da pesquisa realizada em 30 países (European Commission, 2016) e pode ser transposto para o contexto brasileiro de forma agravada, na medida em que não se tem lei nacional no 
país sobre os direitos dos pacientes e a cultura do paternalismo é muito mais arraigada em razão de fatores culturais, sociais e históricos.

Embora o quadro no Brasil seja significativamente inferior ao europeu, correntemente, tem-se dois Projetos de Lei em trâmite que têm como escopo adotar uma lei nacional de direitos dos pacientes, como a seguir será abordado.

\section{Análise das propostas legislativas: PL 5559/16 e PL do Senado $7 / 18$}

Atualmente, encontra-se em trâmite no Congresso Nacional dois Projetos de Lei que podem ser enquadrados como uma proposta de lei nacional de direitos dos pacientes: o PL 5559/16, de autoria dos Deputados Federais, Pepe Vargas - PT/RS, Chico D'Ângelo - PT/RJ, e Henrique Fontana - PT/RS; e o Projeto de Lei do Senado $n^{\circ} 7$, de 2018, de autoria do Senador Pedro Chaves (PSC/MS). O PL 5559/16 dispõe sobre o Estatuto dos Direitos dos Pacientes e o PL do Senado 7/18 trata dos direitos dos pacientes em serviços de saúde; altera o Código Penal para isentar do crime de omissão penalmente relevante a falta de instituição de suporte de vida ou a não realização de tratamento ou procedimento médico recusados; e altera a Lei $n^{\circ} 6.437$, de 20 de agosto de 1977, que configura infrações à legislação sanitária federal.

No que tange ao teor do PL 5559/16, esse se inicia com as definições de autodeterminação; diretivas antecipadas de vontade; representante do paciente; consentimento informado; cuidados paliativos e grupo vulnerável. Quanto aos direitos, o PL 5559/16, prevê os seguintes: médico ou profissional responsável pelos seus cuidados entender que a presença do acompanhante possa acarretar prejuízo à saúde ou à segurança do paciente ou de outrem; direito ao atendimento de qualidade, com profissionais adequados e capacitados; direito à segurança do paciente em procedimentos, ambientes e tratamento; direito à informação sobre seus cuidados em saúde; direito a não ser discriminado; direito à confidencialidade de suas informações pessoais; direito à privacidade; direito de acesso ao prontuário médico e direito de morrer com dignidade, sem sentir dor e de escolher o local de sua morte. Ademais, o PL 5559/16 estabelece as responsabilidades do paciente: os pacientes devem informar suas doenças e tratamentos passados, além do uso de medicamento, a fim de auxiliar os profissionais de saúde no tratamento; e os pacientes devem seguir as instruções, dirimir dúvidas, cumprir regras, respeitar os outros pacientes e os profissionais, entre outros. Por fim, o PL 5559/16 prevê mecanismos de sua efetivação. 
Constata-se que o PL 5559/16 alicerça-se no elenco internacionalmente acordado acerca do rol dos direitos dos pacientes, extraídos dos tratados de direitos humanos, tais como o direito à privacidade, o direito à informação e o direito de não ser discriminado, bem como no modelo do cuidado centrado no paciente (Albuquerque, 2016).

O PL do Senado $7 / 18$, distintamente, não se ancora no consenso internacional acerca de quais são os direitos dos pacientes, consequentemente, estabelece uma série de direitos que não são comumente encontrados em uma lei com tal objeto, tais como: direito de receber relatório escrito contendo informações sobre o diagnóstico e o tratamento indicado; direito de receber prescrições médicas digitadas ou com caligrafia legível; direito de guardar e conservar objetos pessoais durante o período de internação; direito de requerer a gravação de um vídeo que registre os procedimentos dos médicos no centro cirúrgico; e o direito de manter comunicação com pessoas não pertencentes ao serviço de saúde, devendo a direção do serviço de saúde facilitá-la, em especial quando dirigida ao cônjuge, aos pais, responsáveis ou parentes. Por outro lado, o PL do Senado 7/18 é lacunoso quanto a uma série de direitos dos pacientes, exemplificando, o direito do paciente de não ser discriminado; o direito a cuidados em saúde com segurança; o direito ao acompanhante; e o direito à tomada de decisão compartilhada, como se pode verificar no Quadro que coteja ambos os Projetos de Lei que se encontra no apêndice.

Ademais, o PL do Senado 7/18 não estabelece as responsabilidades do paciente, essenciais para a conformação do novo modelo do cuidado centrado no paciente, bem como não estatui mecanismos de efetivação da lei. Ainda, em dissonância com o direito humano do paciente à autodeterminação e a ter sua vontade e preferências respeitadas e com as legislações mais avançadas de outros países (Albuquerque, 2016), o PL do Senado 7/18 não trata das Diretivas Antecipadas de Vontade e Preferências e prevê que a designação de um representante do paciente deve ser feita por instrumento público, o que se revela burocrático, dispendioso e prescindível. Nesse ponto, o PL do Senado 7/18 não contribui para a criação de uma cultura no país, até então inexistente, de elaboração de instrumentos futuros para eventuais situações de incapacidade decisional do paciente.

O PL do Senado $7 / 18$ inova ao ser cotejado com o PL 5999/16 no campo penal ao criminalizar a divulgação por profissional de saúde, sem a expressa autorização do paciente ou de seu representante, de informações relativas à sua condição de saúde, e ao assentar que a falta de instituição de suporte de vida ou a não realização 
de tratamento ou procedimento médico ou odontológico recusados expressamente pelo paciente ou, nos casos em que o paciente não possa expressar sua vontade, por seu representante legal, não é omissão penalmente relevante. Quanto à previsão do crime atinente ao desrespeito à confidencialidade das informações em saúde do paciente, impõe-se a problematização acerca do estímulo à judicialização da medicina, fenômeno pernicioso para o paciente e para o médico. Com relação à omissão penalmente relevante, tendo em conta entendimentos anacrônicos ainda vigentes na jurisprudência brasileira, considera-se que essa previsão do PL é acertada e oportuna. Sendo assim, considerando que o PL do Senado 7/18 traz novos dispositivos quando cotejado com o PL 5999/16, seria oportuna a reflexão sobre a possibilidade de se apresentar uma emenda ao PL 5999/16 a fim de incorporar tais dispositivos.

O sucesso de uma lei de direitos do paciente depende dos direitos enumerados, bem como da interação entre a lei e outras normas legais e deontológicas. Assim, uma lei de direitos dos pacientes consiste num mecanismo de consolidação do modelo do cuidado centrado no paciente e de promoção da nova forma de lidar com as insatisfações e reclamos dos pacientes, qual seja, de forma extrajudicial. As leis dos direitos dos pacientes devem permitir que o paciente possa expressar suas queixas sem precisar recorrer a advogados ou ao Poder Judiciário.

Com efeito, a lei de direito do paciente deve instituir um sistema de queixa de fácil acesso, como o Ombudsman da Saúde e o do Paciente ou os Comissários, o que reduz a litigância e os processos disciplinares contra os profissionais de saúde. Experiências internacionais apontam para a positividade da influência do Ombudsman e de Comissários na alteração dos sistemas de saúde (Flood; May, 2012). Dessa forma, aponta-se para uma lacuna no PL 5559/16 e no PL do Senado 7/18 no sentido de não preverem um serviço ou órgão com incumbência específica para receber e processar as reclamações dos pacientes, bem como para resolvê-las de forma extrajudicial (Albuquerque, 2018).

Por fim, uma lei de direitos do paciente tem o condão de consolidar e promover três concepções: a) tomada de decisão compartilhada; b) cuidado centrado no paciente; c) resolução extrajudicial de conflitos (Health and Social Care Alliance Scotland, 2019), de modo a incrementar a qualidade dos serviços de saúde e o bem-estar do paciente, promovendo a sua autonomia. Sendo assim, tendo em conta o impacto positivo das leis de direitos dos pacientes nos serviços de saúde, no item subsequente problematiza-se o papel do SUS na promoção e defesa de tais direitos. 


\section{A promoção e a defesa dos direitos dos pacientes na es- fera do Sistema Único de Saúde}

Inicialmente, importante diferenciar dois conceitos distintos: o de usuário de serviços de saúde e o de paciente. O usuário é aquele que faz uso de um serviço, podendo ser de saúde ou não, detendo, nessa condição, direitos de usuário. O paciente é aquele que se encontra sob cuidados em saúde e é titular de direito tão somente pelo fato de ser atendido por um profissional de saúde. Desse modo, os direitos do usuário são os direitos que as pessoas têm em relação ao serviços de saúde. Assim, o familiar de um paciente pode ser usuário do serviço de saúde, mas, por óbvio, não é paciente, bem como uma pessoa que é vacinada contra febre amarela em um posto de saúde para viajar, é usuária do serviço, mas não paciente. A noção de paciente é mais ampla, pois abarca além daqueles que fazem uso do SUS, os que apenas recorrem a serviços privados de saúde.

Por outro lado, o termo usuário também é empregado para aquele que faz uso dos serviços de vigilância sanitária prestado pela Agência Nacional de Vigilância Sanitária - ANVISA. Na esfera federal, os direitos dos usuários estão fixados na Lei $n^{\circ} 13.460$, de 23 de junho de 2017, que dispõe sobre participação, proteção e defesa dos direitos do usuário dos serviços públicos da administração pública e define usuário como "pessoa física ou jurídica que se beneficia ou utiliza, efetiva ou potencialmente, de serviço público" (Brasil, 2017). Assim, o direito do usuário regula a relação daquele que faz uso de um serviço com o seu provedor, englobando principalmente direitos referentes a: a) participação social nos processos deliberativo do sistema de saúde, como os referentes à adoção de determinada política pública de saúde e à avaliação do serviço; b) características do serviço prestado, como o atendimento ao usuário, a manutenção de instalações adequadas e o fornecimento de informações sobre o serviço; c) acesso a bens e serviços de saúde, como acesso a leitos, medicamentos e insumos em saúde (Albuquerque, 2016). Por outro lado, os direitos dos pacientes são direitos que emergem do cuidado em saúde e correspondem aos profissionais de saúde com a finalidade de assegurar o bem-estar do paciente e o alívio do seu sofrimento.

Ademais, o usuário pode ou não estar enfermo, a depender da sua condição de saúde, distintamente, o paciente se encontra doente. Essa distinção é crucial para o entendimento das formas de participação nos sistemas de saúde e de acolhimento das reclamações de ambos. Com efeito, a teoria acerca da participação do paciente é distinta da do usuário, na medida em que o fato do paciente se encontrar adoecido 
e muitas vezes sua família também interfere diretamente na sua capacidade de mobilização social e o conduz a ter uma participação mais focada em suas demandas pessoais que emergem das premências da sua condição de saúde. Assim, a participação do paciente é entendida como o processo que permite o paciente de ser parte no curso de tomada de decisão que influencia sua saúde, podendo essa decisão ser no âmbito do cuidado ou de políticas de saúde (Souliotis, 2018).

A participação do usuário, que pode não se apresentar enfermo, caracteriza-se como mais expandida, quer seja pela ausência de limitação de saúde ou pela ausência de uma urgência pessoal que paute sua ação. Dessa forma, a participação do usuário é balizada por uma agenda do cidadão, que se diferencia da agenda do paciente (Albuquerque, 2016), na medida em que a primeira se distingue pelo foco na alocação dos bens e serviços de saúde e na sua organização, ao passo que a agenda do segundo centra-se nos reclamos clínicos, na qualidade da interação com os profissionais de saúde e no atendimento de suas demandas pessoais (Albuquerque, 2016). A partir dessa distinção será abordado em seguida os direitos do usuário e do paciente do âmbito do SUS.

No que toca aos direitos dos usuários na esfera do SUS, a Constituição Federal de 1988 estabelece, em seu art. 196, que a saúde é um direito de todos e dever do Estado, o que se aplica aos usuários e aos pacientes, bem como assenta que a participação da comunidade é um das diretrizes do SUS. A Lei no 8.080, de 19 de setembro de 1990 - Lei Orgânica da Saúde - estabelece a participação da comunidade, enquanto um dos princípios do SUS e, além desse, outros que importam para os usuários, como a "divulgação de informações quanto ao potencial dos serviços de saúde e a sua utilização pelo usuário"; "universalidade de acesso aos serviços de saúde em todos os níveis de assistência"; "integralidade de assistência"; e "igualdade da assistência à saúde" (Brasil, 1990). Quanto ao emprego do termo usuário, a Lei citada apena o utiliza uma vez quando trata da divulgação de informações sobre os serviços de saúde, acima transcrito.

A Lei $n^{\circ} 8.142$, de 28 de dezembro de 1990, que versa sobre a participação da comunidade na gestão do SUS, prevê, em cada esfera de governo, as seguintes instâncias colegiadas: Conferência de Saúde e Conselho de Saúde. A Lei estabelece que a Conferência de Saúde deve ser constituída de modo a ter representação dos vários segmentos sociais, e o Conselho de Saúde como órgão colegiado composto por representantes do governo, prestadores de serviço, profissionais de saúde e usuários. 
A representação dos usuários nos Conselhos de Saúde e Conferências será paritária em relação ao conjunto dos demais segmentos (Brasil, 1990).

Assim, verifica-se que no SUS há instâncias de participação direta da sociedade civil, particularmente de usuários, mas não há alusão a pacientes. Essa exclusão dos pacientes expressa a sua desconsideração como um ator distinto do usuário na esfera dos serviços de saúde, em descompasso com os movimentos internacionais de direitos dos pacientes e as experiências dos sistemas de saúde de variados países. Com efeito, Hashem e Brown investigaram a participação do paciente nos sistemas de saúde e formularam o conceito de "paciente e o envolvimento público - PEP", no contexto do planejamento e do desenvolvimento de serviços de saúde, o que constitui um referencial específico destinado ao estudo e à teorização da participação do Paciente (Hashem; Brown, 2018).

A Carta dos Direitos dos Usuários do SUS, aprovada mediante a Portaria $\mathrm{n}^{\circ} 1.820$, de 13 de agosto de 2009, conforme estudo de Albuquerque (2016), mescla direitos do usuário - direitos relativos à organização dos serviços de saúde e o direito de participar dos conselhos e conferências de saúde - e direitos de paciente, como o direito ao consentimento informado e ao acompanhante (Brasil, 2009).

Embora a Carta seja relevante para se iniciar a criação de uma consciência acerca dos direitos dos pacientes, o fato de misturar direitos de natureza distintas, de não ser uma lei, de não ter criado um mecanismo de implementação e de não se aplicar a serviços privados de saúde sem vínculo com o SUS, concorre para não ser um instrumento eficaz na criação de uma cultura de respeito dos direitos do paciente no Brasil. Como se viu no Relatório da União Europeia, atualmente, após décadas da adoção de leis de direitos do paciente na maior parte dos países do continente europeu, ainda se enfrenta obstáculos para tornar os direitos dos paciente conhecidos da ampla maioria das pessoas e para disseminar o seu papel central na melhora da qualidade dos serviços de saúde.

Em relação ao gestor nacional do SUS, na atual estrutura do Ministério da Saúde, o usuário é mencionado apenas quando se trata da competência da Secretaria-Executiva para gerir e assegurar o cumprimento de decisões judiciais no âmbito do Ministério da Saúde (Brasil, 2018).

No que concerne aos direitos dos pacientes no âmbito do SUS, esses não foram priorizados em sua história. De acordo com Escorel, Nascimento e Edler, as origens 
da Reforma Sanitária e do SUS remontam a movimentos, da década de sessenta, críticos ao foco na medicina individual e defensores da política de saúde com ênfase na coletividade. Na década de oitenta, o movimento da Reforma Sanitária, ao contestar o modelo vigente de serviços de saúde, propôs alternativas fundamentadas em um sistema de saúde democrático e universal, bem como alicerçado na participação social. (Escorel; Nascimento; Edler, 2005). No Relatório Final da $8^{a}$ Conferência Nacional de Saúde (1986), realizada em 1986, que assentou as bases do SUS, há a expressão da preocupação em torno do escassez de participação popular no processo de construção de políticas públicas e no controle dos serviços de saúde.

Ainda, o Relatório enfatiza a importância de "estimular a participação popular nos núcleos decisórios" (Conferência Nacional de Saúde, 1986). Assim, o movimento da Reforma Sanitária incorporou aqueles que faziam uso dos serviços de saúde, tão somente pela perspectiva da participação cidadã, contemplando a agenda do cidadão. Em consequência, deixou de lado a agenda do paciente e dos seus direitos (Albuquerque, 2016). A ênfase na perspectiva coletiva e a crítica ao modelo de medicina individual podem ser fatores que expliquem a ausência do paciente, como sujeito de direitos do SUS.

Como exemplo, na Lei $n^{\circ} 8.080$, de 1990 , há a alusão ao paciente somente duas vezes ao tratar do Subsistema de Atendimento e Internação Domiciliar. Contudo, pode-se asseverar que há dois princípios atinentes ao paciente: o referente à preservação da autonomia das pessoas na defesa de sua integridade física e moral e o direito à informação, às pessoas assistidas, sobre sua saúde (Brasil, 1990).

Importante ressaltar que os direitos dos pacientes não são apenas endereçados àqueles que se encontram assistidos pelo SUS, mas se dirigem a todas as pessoas que se encontram sob cuidados em saúde, independentemente da natureza do serviço, se público ou privado, ou se presente ou não relação de consumo. Dessa forma, os direitos dos pacientes previstos na Carta do SUS não dizem respeito àqueles que são atendidos por serviços ou profissionais sem vínculos com o SUS. De acordo com a Lei Orgânica da Saúde, os serviços privados de saúde devem observar os princípios éticos e as normas expedidas pelo órgão de direção do SUS, o Ministério da Saúde, quanto às condições para seu funcionamento (Brasil, 1990). Assim, o espectro de atribuição do Ministério da Saúde relativo aos serviços privados de saúde abarca tão somente as condições para funcionarem, o que não envolve a prescrição de direitos dos pacientes. 
Na esfera do Ministério da Saúde, não há nenhum órgão com competência para tratar dos direitos dos pacientes (Brasil, 2018), isto é, de políticas e programas destinados à conscientização da sociedade em torno da sua importância, aos modos de promover a sua capilaridade nos serviços de saúde e ao recebimento e ao processamento de reclamações de pacientes. Quanto a tal ponto, sublinha-se que a Ouvidora-geral do SUS não tem a incumbência de resolver as queixas dos pacientes ou de coordenar mecanismos com essa finalidade, como o Ombudsman do Paciente, Comissões ou serviços com tal fim. No Decreto que estrutura o Ministério da Saúde, o termo paciente apenas é empregado uma única vez quando trata do programa nacional de segurança do paciente (Brasil, 2018).

Os direitos dos pacientes e a saúde pública compartilham aproximações, como a busca por resultados positivos em saúde, e distanciamentos, como o foco individual, dos direitos dos pacientes, e populacional, da saúde pública ou coletiva, a depender da abordagem. Com efeito, os direitos dos pacientes dizem respeito ao tratamento e ao alívio do sofrimento de uma pessoa e a saúde pública concerne àquilo que o Estado e as sociedades fazem coletivamente, para assegurar as condições de vida saudável da população (Peled-Raz, 2017).

Desse modo, sustenta-se que a perspectiva individual, do paciente, e a coletiva, da saúde pública ou coletiva, podem ser convergentes e não se excluem. Nesse sentido, o mesmo sistema de saúde que tem a finalidade de assegurar as condições de saúde da população também tem a obrigação de garantir os direitos dos pacientes. Igualmente, sublinha-se que os direitos dos pacientes desenvolvem uma função singular nos sistemas de saúde. Na atualidade, a complexidade dos procedimentos e intervenções médicas advinda das novas tecnologias; as mudanças demográficas que ocasionam o envelhecimento da população; e as consequências para os sistemas de saúde do aumento das doenças crônicas impõem ao Estado novas formas de lidar com os cuidados em saúde, as quais devem se ancorar em estratégias que envolvam a participação dos pacientes e a salvaguarda de seus direitos (European Commission, 2016).

Particularmente, na esfera do SUS, compete ao Ministério da Saúde coordenar a implementação da lei nacional de direitos dos pacientes, por meio de um órgão específico em sua estrutura e de mecanismos de recebimento de reclamação descentralizados, mas com uma coordenação única. Ademais, a lei de direitos dos pacientes, por meio do modelo do cuidado centrado no paciente que o baliza, pode contribuir 
para que as políticas e programas do SUS não foquem no doença, mas sim no paciente, enquanto ator central do cuidado em saúde e titular de direitos.

Com efeito, Hunt e Backman (2009) apontam que os sistemas de saúde devem incorporar uma visão holística e centrada na pessoa. Assim, conclui-se que o SUS, além de garantir o direito à saúde da população, tem um papel central e insubstituível na formulação e na implementação de uma política nacional dos direitos dos pacientes, contando com órgãos e orçamento específico para tal desiderato. A lei nacional de direitos dos pacientes é essencial, mas sem a política pública e a sua estruturação institucional correlatas, a lei não passará de apenas uma medida legislativa sem força para promover alterações efetivas na prática do cuidado em saúde.

A visão reducionista, que predomina no Brasil no sentido de desconsiderar as especificidades da condição de paciente não contribui para a expansão de uma cultura de direitos no SUS. O usuário e o paciente são atores centrais e distintos do SUS, logo, ambos devem ter seus direitos previstos em lei, o que já ocorreu com os usuários na esfera federal, mas, não se verifica o mesmo com relação aos pacientes, haja vista que não há uma lei no Brasil sobre direitos dos pacientes.

\section{Considerações Finais}

As leis de direitos dos pacientes são instrumentos essenciais para a alteração da cultura dos cuidados em saúde no Brasil. Atualmente, a relação entre profissional de saúde e paciente se encontra deteriorada em razão da desconfiança provocada pela judicialização exacerbada da ocorrência de erros na saúde; pela formação excessivamente tecnicista dos profissionais em saúde em contraposição à aprendizagem de conteúdos humanistas, inclusive do modelo do cuidado centrado no paciente; e pelas questões relacionadas à alocação de leitos e de medicamentos e aos planos privados de saúde, que não foram objeto deste artigo.

Desse modo, tem-se um panorama complexo na área dos cuidados em saúde que precisa ser enfrentado pelas organizações representativas de pacientes, profissionais e provedores de serviços de saúde e seus usuários. A busca por melhores resultados em saúde é coletiva e abarca todos os setores da sociedade brasileira, contudo, a baixa consciência acerca da importância dos direitos dos pacientes compromete o uso de tal instrumento como uma ferramenta potente a ser usada para a melhora da saúde de todos. A aprovação da lei de direitos dos pacientes em conjunto 
com a adoção de política pública e com a sua institucionalização no SUS, por meio de serviços destinados aos pacientes, é fator central para que o Brasil possa se alçar a outro patamar quanto à qualidade nos cuidados em saúde e ao respeito aos direitos humanos nessa esfera.

Para tanto, o papel do SUS é nodal, na medida em que uma política pública sobre direitos dos pacientes demanda sua estruturação nacional e seu espraiamento nas esferas locais, coordenada pelo Ministério da Saúde. Desse modo, o endosso da necessidade de se contar com uma lei de direitos dos pacientes no Brasil se correlaciona com a assunção pelo órgão gestor nacional do SUS da responsabilidade de proteger os direitos dos pacientes por meio de medidas efetivas. Reconhece-se que essa mudança propugnada corresponde a um caminho tortuoso e de longo prazo, porém, o pontapé inicial há que ser dado mediante a aprovação do Projeto de Lei n 5559/16.

\section{Referências}

ALBUQUERQUE, A. Direitos Humanos dos Pacientes. Curitiba: Juruá, 2016.

AMERICAN INSTITUTES FOR RESEACHER. Placing Patients the Center of Health Care. Acessível em: https://www.air.org/resource/placing-patients-centerhealth-care.

ARGENTINA. Derechos del paciente, historia clínica y consentimiento informado. Acessível em: http://servicios.infoleg.gob.ar/infoleglnternet/anexos/160000164999/160432/norma.htm.

BRASIL. Decreto $n^{\circ} 8.901$, de 10 de novembro de 2018. Disponível em: http://www. planalto.gov.br/ccivil_03/_Ato2015-2018/2016/Decreto/D8901.htm. Acesso em: 12 abril 2019.

. Lei $N^{0} 13.460$, de 26 de Junho de 2017. Disponível em: http://www.planalto.gov.br/ccivil_03/_ato2015-2018/2017/lei//13460.htm. Acesso em: 12 abril 2019.

Portaria $\mathrm{n}^{\circ} 1.820$, de 13 de agosto de 2009. Disponível em: http://bvsms. saude.gov.br/bvs/saudelegis/gm/2009/prt1820_13_08_2009.html. Acesso em: 12 abril 2019.

Lei $n^{\circ}$ 8.142, de 28 de dezembro de 1990. Disponível em: http://www. planalto.gov.br/ccivil_03/leis/L8142.htm. Acesso em: 12 abril 2019.

COHEN, J; EZER, T. Human rights in patient care: A theoretical and practical framework. Health and Human Rights 2013; 15(2): E7-E19.

EQUADOR. Ley de derechos y amparo del paciente. Ley 77. Acessível em: https:// www.salud.gob.ec/wp-content/uploads/downloads/2014/09/Normativa-Ley-deDerechos-y-Amparo-del-Paciente.pdf. Acesso em: 8 abril 2019. 
ESCOREL, S; NASCIMENTO, DR; EDLER, FC. As origens da Reforma Sanitária e do SUS. In: LIMA, NT; GERSCHMAN, S; EDLER, FC; SUÁREZ, JM. Saúde e Democracia: história e perspectivas do SUS. Rio de Janeiro: Fiocruz, 2005: 59-83.

EUROPEAN COMMISSION. Patients' Rights in the European Union Mapping eXercise. Luxembourg: Publications Office of the European Union, 2016.

FLOOD, CM; MAY, K. A patient charter of rights: how to avoid a toothless tiger and achieve system improvement. Canadian Medical Association Journal 2012, 184 (14): 89-96.

HANS TRIER, A; VALDERAS, JM; WENSING, M; MARTIN, HM; EGEBART, J. Involving patients in patient safety programmes: A scoping review and consensus procedure by the LINNEAUS collaboration on patient safety in primary care. Eur J Gen Pract 2015; 21(sup1): 56-61.

HASHEM, F; BROWN, PR. Decision making in NICE single technological appraisals: How does NICE incorporate patient perspectives? Health Expectations 2018; 21:128-137.

HEALTH AND SOCIAL CARE ALLIANCE SCOTLAND. Being human: a human rights based approach to health and social care in Scotland. Acessível em: https://www. alliance-scotland.org.uk/wp-content/uploads/2017/11/ALLIANCE-BeingHuman-publication-2017-1.pdf.

HEALY, J. Patients as regulatory actors in their own health care. Acessivel em: http:// press-files.anu.edu.au/downloads/press/n2304/pdf/ch34.pdf.

HUNT, P; BACKMAN, G. Health Systems and the right to the highest attainable standard of heath. In: CLAPHAM, A; ROBINSON, M. Realizing the Right to Health. Zurique: Ruffer \& Rub, 2009: 40-60.

JUN, HS. Concept analysis of patient rights. Korean Journal of Adult Nursing 2019; 31 (1):89-99.

MALIK, M. Advocacy in nursing - a review of the literature. J Adv Nurs 1997; 25 (1):130138.

MEESALA, A; PAUL, J. Service quality, consumer satisfaction and loyalty in hospitals: Thinking for the future. Journal of Retailing and Consumer Services. 2018; 40: 261-269.

$8^{\text {a }}$ CONFERENCIA NACIONAL DE SAÚDE. Relatório Final. Acessível em:

http://bvsms.saude.gov.br/bvs/publicacoes/8_conferencia_nacional_saude_relatorio_fin al.pdf.

PARANHOS, D. Direitos Humanos dos Pacientes Idosos. Rio de Janeiro: Lúmen Juris, 2018.

PELED-RAZ, M. Human rights in patient care and public health - a common ground. Public Health Review 2017; 38:29. 
SCHYVE, PM. Patient Rights and Organization Ethics: The Joint Commission Perspective. Bioethics. 1996: 13-20.

SOULIOTIS, $\mathrm{K}$ et al. Assessing patient Organizations participation in health policy: a comparative study in France and Italy. International Journal of Health Policy and Management 2018; 7(1): 48-58.

UNITED NATIONS. H.R.4449 - Patient Self Determination Act of 1990. Acessível em: https://www.congress.gov/bill/101st-congress/house-bill/4449.

URUGUAY. Ley No 18.335. Pacientes y usuarios de los servicios de salud se establecen sus derechos y obligaciones. Acessível em:

https://legislativo.parlamento.gub.uy/temporales/leytemp1589866.htm.

Recebido: 11/09/2019. Aprovado: 12/10/2019. 


\section{Apêndice}

\section{QUADRO COMPARATIVO}

\begin{tabular}{|c|c|}
\hline PL 5559/16 & PL DO SENADO $7 / 18$ \\
\hline $\begin{array}{l}\text { Art. } 6^{\circ} \text {. O paciente tem o direito de } \\
\text { indicar livremente um representante } \\
\text { em qualquer momento de seus } \\
\text { cuidados em saúde, por meio de } \\
\text { registro em seu prontuário. }\end{array}$ & \\
\hline $\begin{array}{l}\text { Art. } 7^{\circ} \text {. O paciente tem o direito de } \\
\text { contar com um acompanhante em } \\
\text { consultas e internações. }\end{array}$ & \\
\hline $\begin{array}{l}\text { Art. } 8^{\circ} \text {. O paciente tem direito de } \\
\text { ter acesso a cuidados em saúde de } \\
\text { qualidade, no tempo oportuno, e de } \\
\text { ser atendido em instalações físicas } \\
\text { limpas e adequadas. }\end{array}$ & \\
\hline $\begin{array}{l}\text { Art. } 9^{\circ} . \text { O paciente tem o direito } \\
\text { de que sua segurança seja } \\
\text { assegurada, oque implicaambiente, } \\
\text { procedimentos e insumos seguros. }\end{array}$ & \\
\hline $\begin{array}{l}\text { Art. } 10^{\circ} \text {. O paciente tem o direito } \\
\text { de não ser tratado com distinção, } \\
\text { exclusão, restrição ou preferência } \\
\text { baseados em raça, cor, religião, } \\
\text { enfermidade, deficiência, } \\
\text { orientação sexual ou identidade de } \\
\text { gênero, origem nacional ou étnica, } \\
\text { renda, de modo que provoque } \\
\text { restrições em seus direitos. }\end{array}$ & \\
\hline
\end{tabular}


Art. 11. O paciente tem o direito de envolver-se ativamente em seus cuidados em saúde, participando da decisão sobre seus cuidados em saúde e do plano terapêutico.

Art. 12. O paciente tem o direito à informação sobre sua condição de saúde, o tratamento e eventuais alternativas, os riscos e benefícios dos procedimentos, e os efeitos adversos dos medicamentos.

Art. $2^{\circ}$

II-direito dereceberesclarecimentos sobre todos os aspectos relativos a sua condição de saúde e de ter acesso irrestrito ao conteúdo de seu prontuário. III - direito de receber informações claras e objetivas sobre as questões referentes à propedêutica, ao diagnóstico e ao tratamento.

V-direito de receber relatório escrito contendo informações sobre o diagnóstico e o tratamento indicado.

Art. $2^{\circ}$

Art. 13. O paciente tem o direito de VI - direito d e ser informado se o tratamento, o medicamento e o método diagnóstico são experimentais.

ser previamente informado quando $o$ tratamento proposto for experimental ou fizer parte de pesquisa.

Art. 14. O paciente tem direito ao consentimento informado sem coerção ou influência indevida, salvo em situações de risco de morte em que esteja inconsciente. 


\begin{abstract}
Art. 15. O paciente tem direito à confidencialidade das informações sobre seu estado de saúde, tratamento e outras de cunho pessoal, mesmo após sua morte, salvo as exceções previstas em lei.

Art. 16. O paciente tem direito de consentir ou não com a revelação de informações pessoais para terceiros não previamente autorizados, incluindo familiares, exceto quando houver determinação legal.
\end{abstract}

Art. 17. O paciente tem o direito de ter vida privada respeitada quando submetido a cuidados em saúde, o que compreende:

I - o direito de ser examinado em lugar privado, salvo em situações de emergência ou de cuidados intensivos; II - o direito de recusar qualquer visita; e III - o direito de consentir ou não a presença de estudantes e profissional de saúde estranho aos seus cuidados em saúde.

Art. 18. O paciente tem direito de buscar segunda opinião ou parecer de outro profissional ou serviço sobre seu estado de saúde ou procedimentos recomendados,
Art. $2^{\circ}$

VIII - direito à confidencialidade das informações referentes ao seu estado de saúde.
Art. $2^{\circ}$

$X$ - direito de receber ou de se recusar a receber, no local de internação, pessoas não diretamente envolvidas na assistência à sua saúde ou no funcionamento do serviço de saúde.

XI - respeito à privacidade e à intimidade durante o período em que estiver sob os cuidados do serviço de saúde.

\section{Art. $2^{\circ}$}

VII - liberdade de consultar outros profissionais de saúde, além daqueles que o estiverem assistindo, a fim de obter outra opinião ou esclarecimento a respeito do seu diagnóstico, prognóstico ou tratamento. 


\begin{tabular}{|c|c|}
\hline $\begin{array}{l}\text { Art. 19. O paciente tem o direito de ter } \\
\text { acesso ao seu prontuário médico, sem } \\
\text { necessitar apresentar justificativa. }\end{array}$ & $\begin{array}{l}\text { Art. } 2^{\circ} \\
\text { II - direito de receber esclarecimentos } \\
\text { sobre todos os aspectos relativos } \\
\text { a sua condição de saúde e de ter } \\
\text { acesso irrestrito ao conteúdo de seu } \\
\text { prontuário. }\end{array}$ \\
\hline \multicolumn{2}{|l|}{$\begin{array}{l}\text { Art. } 20 . \text { O paciente tem o direito de ter } \\
\text { suas diretivas antecipadas de vontade } \\
\text { respeitadas pela família e pelos } \\
\text { profissionais de saúde. }\end{array}$} \\
\hline $\begin{array}{l}\text { Art. 21. O paciente tem o direito de } \\
\text { morrer com dignidade, livre de dor e } \\
\text { de escolher o local de sua morte. }\end{array}$ & $\begin{array}{l}\text { Art. } 2^{\circ} \\
\text { IX - acesso a cuidados paliativos } \\
\text { adequados ao alívio do sofrimento } \\
\text { e de sintomas físicos e psíquicos } \\
\text { decorrentes de doenças ou agravos } \\
\text { à saúde, independentemente da } \\
\text { perspectiva de cura ou de mudança } \\
\text { na evolução do quadro clínico. }\end{array}$ \\
\hline & $\begin{array}{l}\text { Art. } 2^{\circ} \\
\text { IV - direito de receber prescrições } \\
\text { médicas digitadas ou com caligrafia } \\
\text { legível. }\end{array}$ \\
\hline & $\begin{array}{l}\text { Art. } 2^{\circ} \\
\text { XII - direito de guardar e conservar } \\
\text { objetos pessoais durante o período de } \\
\text { internação. }\end{array}$ \\
\hline & $\begin{array}{l}\text { Art. } 2^{\circ} \\
\text { XIII - direito de requerer a gravação } \\
\text { de um vídeo que registre os } \\
\text { procedimentos dos médicos no centro } \\
\text { cirúrgico. }\end{array}$ \\
\hline
\end{tabular}




\begin{tabular}{|l|l|}
\hline & Art. $2^{\circ}$ \\
& XIV - direito de manter comunicação \\
& com pessoas não pertencentes ao \\
serviço de saúde, devendo a direção \\
do serviço de saúde facilitá-la, em \\
especial quando dirigida ao cônjuge, \\
aos pais, responsáveis ou parentes.
\end{tabular}

